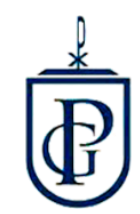

Veritas Et Scientia

Vol. 7, N $1,866-876$

Enero - Junio del 2018.

ISSN $2307-5139$

\title{
INFLUENCIA DE LOS ESPACIOS PÚBLICOS DEL CAMPUS CAPANIQUE SL-02 EN LA FORMACIÓN UNIVERSITARIA DE LOS ESTUDIANTES DE LA FAU-UPT- 2017
}

\author{
INFLUENCE OF THE PUBLIC SPACES OF CAMPUS CAPANIQUE SL-02 IN THE UNIVERSITY FORMATION \\ OF THE STUDENTS OF THE FAU-UPT- 2017
}

Catherine Alessandra Almonte Durand ${ }^{1}$

\section{RESUMEN}

El objetivo de este estudio es determinar de qué manera los espacios públicos del Campus Capanique SL-02 influyen en la formación universitaria de los estudiantes de la Facultad de Arquitectura y Urbanismo de la Universidad Privada de Tacna, considerando el factor Infraestructura como un alcance predominante perteneciente al indicador bienestar institucional, potenciando dentro de este factor la importancia del grado de excelencia en calidad paisajista que debiera existir en cada espacio físico. La investigación es del tipo pura, con un diseño explicativo. La población de estudio estuvo conformada por los estudiantes de la Facultad de Arquitectura y Urbanismo. El tamaño de muestra fue de 200 estudiantes elegidos aleatoriamente. El instrumento fue la encuesta validada con el coeficiente de Alpha de Cronbach, y aplicada a la muestra representativa. Los resultados fueron procesados y analizados con el programa estadístico SPSS.Los resultados se expresan en las tablas resumen donde se establecen los niveles de comportamiento que presenta cada variable. Los resultados permiten establecer que los espacios públicos tienen influencia significativa sobre la formación universitaria en la Facultad de Arquitectura y urbanismo. En conclusión, se puede establecer que los espacios públicos del Campus SL-02 de la Facultad de Arquitectura y Urbanismo condiciona la calidad de la formación de los estudiantes de la carrera de Arquitectura.

Palabras Clave: Espacio Público, calidad, formación universitaria, valoración paisajista.

\footnotetext{
${ }^{1}$ Maestro en arquitectura paisajista
} 


\begin{abstract}
The objective of this study is to determine how the public spaces of Campus Capanique SL-02 influence the university education of students of the Faculty of Architecture and Urbanism of the Private University of Tacna, considering the Infrastructure factor as a predominant scope belonging to the institutional welfare indicator, promoting within this factor the importance of the degree of excellence in landscape quality that should exist in each physical space. The research is of the pure type, with an explanatory design. The study population was made up of students from the Faculty of Architecture and Urbanism. The sample size was 200 students randomly chosen. The instrument was the survey validated with the Cronbach's Alpha coefficient, and applied to the representative sample. The results were processed and analyzed with the statistical program SPSS. The results are expressed in summary tables where the levels of behavior presented by each variable are established. The results allow establishing that public spaces have significant influence on university education in the Faculty of Architecture and Urbanism. In conclusion, it can be established that the public spaces of Campus SL02 of the Faculty of Architecture and Urbanism condition the quality of the training of the students of the Architecture career.
\end{abstract}

Keywords: Public space, quality, university education, landscape assessment

\title{
INTRODUCCIÓN
}

En un mundo contemporáneo con formas de vida cada vez más globalizadas, cuando se habla de universidad, se identifica rápidamente como protagonista del tema en cuestión, a personas jóvenes en su mayoría, que se caracterizan por la exposición a múltiples factores que influencian su forma de vida. Es de vital importancia resaltar, la etapa de vulnerabilidad en la que se encuentran el mayor porcentaje de estudiantes universitarios (entre 18 y 22 años), los cuales psicológicamente se encuentran en la etapa final de formación personal. Un estudiante universitario en el desenvolvimiento de su vida diaria permanece dentro del claustro universitario aproximadamente el $70 \%$ del día, realizando ejercicios vivenciales, sencillos y prácticos, los cuales se ven potenciados en el ambiente o espacio físico indicado que brinde características de confort e invite al usuario a permanecer en ellos. Los espacios públicos representan, áreas físicas que de organizarse de la manera adecuada ayuda a combatir el grado de estrés (debilidades) y potenciar factores de resiliencia (fortalezas), convirtiéndolos en profesionales que cultivan habilidades para la vida, es importante potenciar el intercambio de experiencias con los compañeros de grupo, experiencias que no deberían limitarse únicamente a ser compartido en aulas o pasillos, sino más bien en espacios públicos exteriores. Los motivos por los que es importante contar con una valoración paisajista adecuada, o proponer mejorar la calidad del paisaje con la que cuentan las universidades, a través por lo menos de la presencia de más áreas verdes pueden mejorar el desempeño mental de los estudiantes, influenciando positivamente en la formación universitaria que reciben. La existencia de universidades que han demostrado brindar formación de calidad en consideración a estos factores e indicadores (formación universitaria integral), como es el caso de la Universidad Privada de Tacna y como lo declara en su política de calidad, en su constante preocupación por preparar a sus profesionales no solo en el ámbito académico sino en el ámbito personal. 
Sin embargo en la actualidad la falta del uso de sus espacios públicos, y la poca valoración paisajista con la que cuentan los mismos citando la poca consideración al paisaje (naturaleza) que ayuda en general a tener más felicidad y mejorar las tareas mentales, son el principal objetivo de esta investigación, la cual comprueba en su desarrollo la importancia de brindar la atención necesaria a este tema, quedando así comprobada la importancia de la estrecha relación que mantiene con la formación universitaria.

La calidad del espacio público es un tema crucial, con respecto a ello "Segovia y Jordán (2005) afirman que la calidad del espacio público se podrá evaluar sobre todo por la intensidad y la calidad de las relaciones sociales que facilita, por su capacidad de acoger y mezclar distintos grupos y comportamientos, y por su oportunidad para estimular la identificación simbólica, la expresión y la integración cultural".

Gonzales, P (2016) concluye que al aplicar las teorías estudiadas del uso y la apropiación del espacio público existe alta relación entre estas y las actividades sociales del sector estudiado. Marín, L (2016) aplica el principio del espacio urbano y su impacto sobre la ciudad, a un espacio que utiliza la misma estructura, la universidad como ciudad y el espacio público como tal, así ambos concluyen en que las presencias de dichos espacios públicos contribuyen con la mejora de la calidad de vida de los estudiantes potenciando la realización de distintas actividades. Salcedo, JC (2012) refiere que la creación de un espacio arquitectónico para el desarrollo de la actividad universitaria no es fácil; requiere tanto espacios construidos de calidad funcional y formal, como espacios urbanos adecuados. Burneo, L (2011). Propone indagar el vínculo "ciudadano-ciudad" que se establece entre los habitantes y el espacio público, la cual se ve reflejada en las formas de apropiación. Afirma que los espacios públicos posibilitan el encuentro y la interacción entre personas, contribuyendo a la tolerancia entre grupos sociales diversos y a la construcción del sentido de vida colectiva. Jover y Gozalvez sitúan su análisis como una clave para determinar la continuidad o discontinuidad con el mundo, a partir de dos de los principales debates de la historia contemporánea en torno a la universidad y la educación, que confluyen en la figura central del pragmatismo pedagógico. De la confrontación de estas ideas, se extraen una relación de propuestas con respeto a la universidad de hoy, y que esta debería cumplir con las siguientes características: 1) instituir el equilibrio entre las ciencias y las humanidades, 2) recrear su dimensión comunitaria como un espacio público favorecedor y 3) reinterpretar el pragmatismo, insistiendo en los aspectos axiológicos y políticos del mismo, su compromiso con la igualdad y los valores democráticos.

El estudiante universitario dentro del campus se define como actor principal dentro de la institución. Es el habitante cuya primordial característica se define como la permanencia esporádica o mantenerse de paso por el lugar, la existencia de los mismos afirma la existencia de la universidad, es por ello la importancia del aporte que realizan los espacios físicos dentro de la formación que recibe el usuario. Jan Gehl dedica casi el texto completo a la importancia de la dinámica social para identificación de la calidad de un espacio determinado para comunidad humana que lo habita.

Cualquier espacio exterior o interior considerado público, o de un gran universo debiera poder mostrar arquitectónicamente el tipo de organización que utiliza. Se define como organización espacial a la capacidad de situarse en el espacio determinado de la posición que uno ocupa en relación a referencias externas, ordenar correctamente los diferentes elementos de un conjunto, apreciar las relaciones que existen entre uno y otro elemento, los objetos de un punto de vista topológico, proyectivo y euclidiano (F. Ching, 2010). José María Montaner (1997) menciona: "el lugar viene definido por sustantivos, por las cualidades de las cosas y por los elementos, por los valores simbólicos e históricos; es ambiental y está relacionado lógicamente con el cuerpo humano", por lo tanto, el espacio físico delimitado para una función específica, permitirá al diseño arquitectónico ser y permanecer, ya sea como en este caso para convertirse en un espacio público de una tipología determinada ya sea un parque, una plaza, etc. El uso de este espacio estará determinado por la potencialidad que tenga el diseño que se realice, no nos limitamos solo a existir sino a permanecer porque se tiene una función, esa es la clave de la arquitectura. Giddens (1995: 98) sugiere que "las 
rutinas de vida cotidiana son fundamentales aun para las formas más elaboradas de organización societaria". El paisaje, es a la vez, una realidad física y la representación que culturalmente nos hacemos de ella. Es al mismo tiempo, significante y significado, el continente y el contenido, la realidad y la ficción. (Nogue, J. 2008)

Los objetivos de calidad paisajística se refieren a líneas estratégicas de actuación, tomando en cuenta la participación pública, para establecer los objetivos se deber tener en cuenta fundamentalmente dos aspectos, el valor del paisaje y los conflictos a los que se ve sometido"

\section{OBJETIVOS}

- Establecer en qué medida se relaciona la organización espacial del Campus Capanique SL-02 con la formación universitaria de la Facultad de Arquitectura y Urbanismo de la Universidad Privada de Tacna.

- Establecer en qué medida se relaciona el uso y apropiación de espacios del Campus Capanique SL-02 con la formación universitaria de la Facultad de Arquitectura y Urbanismo de la Universidad Privada de Tacna.

- Determinar en qué medida se relaciona la valoración y calidad del paisaje del Campus Capanique SL-02 con la formación universitaria de la Facultad de Arquitectura y Urbanismo de la Universidad Privada de Tacna.

\section{METODOLOGÍA}

Tipo de investigación básica, descriptiva y explicativa, no experimental. El ámbito de estudio del presente trabajo de investigación es la Universidad Privada de Tacna y comprende el Campus Capanique SL-02 ubicado en el distrito de Pocollay de Tacna. La unidad de estudio fue la población estudiantil que pertenece a la Facultad de Arquitectura y Urbanismo de la Universidad Privada de Tacna y tuvo un tamaño de 200 estudiantes.

La validación de los instrumentos se realizo a través de la prueba de confiabilidad del estadístico Alpha de Cronbach, con un valor final de 0.938 .

\section{RESULTADOS}

La información procesada se presenta según el siguiente orden:

a) Información sobre los espacios públicos

b) Información sobre formación universitaria

Tabla 1: Espacio público

\begin{tabular}{|c|c|c|}
\hline Indicador & Media & Desv. típica \\
\hline Espacio público & 3.64 & 0.39 \\
\hline Indicador & Sumatoria & \\
\hline Espacio público & 14.55 & \\
\hline Ítems & Media & Desv. Típica \\
\hline $\begin{array}{l}\text { La relación espacial existente dentro del espacio } \\
\text { público es: }\end{array}$ & 3.78 & .681 \\
\hline $\begin{array}{l}\text { Los elementos conectores con los que cuenta el } \\
\text { espacio público para enlazarse con otro espacio } \\
\text { público son: }\end{array}$ & 3.27 & .432 \\
\hline $\begin{array}{l}\text { El tipo de circulación interior (recorridos peatonales) } \\
\text { con la que cuenta el espacio público es: }\end{array}$ & 3.54 & .307 \\
\hline La percepción de la función dada al espacio es: & 3.96 & .128 \\
\hline
\end{tabular}


Los resultados de la Tabla 1 representan el comportamiento del indicador espacios públicos, donde se observa que la sumatoria de las medias de los ítems es 14.55 , que se encuentra comprendida en la escala de (11-16), lo cual significa que los estudiantes expresan que los espacios públicos que caracterizan a la Facultad de Arquitectura y Urbanismo, son adecuados, lo cual implica que el campus Capanique SL-02 de la Universidad Privada de Tacna, cumple con las condiciones fundamentales para la formación de los estudiantes.

Los resultados de la Tabla 2 representan el comportamiento del indicador actividades sociales, donde se observa que la sumatoria de las medias de los ítems es 11.39 , que se encuentra comprendida en la escala de (7-12), lo cual significa que los estudiantes expresan que las actividades sociales que se realizan en los espacios públicos de la Facultad de Arquitectura y Urbanismo, son adecuados, lo cual implica que en el campus Capanique SL-02 de la Universidad Privada de Tacna, cumple con las condiciones fundamentales para la formación de los estudiantes.

Tabla 2: Actividades sociales

\begin{tabular}{|c|c|c|}
\hline Indicador & Media & Desv. típica \\
\hline Actividades sociales & 3.79 & 0.50 \\
\hline Indicador & Sumatoria & \\
\hline Actividades sociales & 11,39 & \\
\hline Ítems & Media & Desv. Típica \\
\hline $\begin{array}{l}\text { En qué nivel desarrollan } \\
\text { actividades NECESARIAS }\end{array}$ & 3.88 & .642 \\
\hline $\begin{array}{l}\text { En qué nivel desarrollan } \\
\text { actividades OPCIONALES }\end{array}$ & 3.77 & .232 \\
\hline $\begin{array}{l}\text { En qué nivel desarrollan } \\
\text { actividades NETAMENTE } \\
\text { SOCIALES }\end{array}$ & 3.74 & .637 \\
\hline
\end{tabular}

Los resultados de la Tabla 3 representan el comportamiento del indicador relaciones sociales, donde se observa que la sumatoria de las medias de los ítems es 13.45 , que se encuentra comprendida en la escala de (11-16), lo cual significa que los estudiantes expresan que las relaciones sociales que se realizan en los espacios públicos de la Facultad de Arquitectura y Urbanismo, son adecuados, lo cual implica que el campus Capanique SL-02 de la Universidad Privada de Tacna, cumple con las condiciones fundamentales que exige la calidad en la formación de los estudiantes.

Tabla 3: Condiciones para relaciones sociales

\begin{tabular}{|c|c|c|}
\hline Indicador & Media & Desv. típica \\
\hline Relaciones sociales & 3.36 & 0.38 \\
\hline Indicador & Sumatoria & \multicolumn{1}{|}{} \\
\hline Relaciones sociales & 13.45 & \\
\cline { 1 - 2 }
\end{tabular}

Los resultados de la Tabla 4 representan el comportamiento del indicador atributos biofísicos, donde se observa que la sumatoria de las medias de los ítems es 18.48 , que se encuentra comprendida en la escala de (16-24), lo cual significa que los estudiantes expresan que los atributos biofísicos de los espacios públicos de la Facultad de Arquitectura y Urbanismo, son adecuados, lo cual implica que el campus Capanique SL-02 de la Universidad Privada de Tacna, cumple con las condiciones fundamentales que exige la calidad en la formación de los estudiantes. 
Tabla 4: Atributos biofísicos

\begin{tabular}{|c|c|c|}
\hline Indicador & Media & Desv. típica \\
\hline Atributos biofísicos & 3.08 & 0.35 \\
\hline Indicador & Sumatoria & \multirow{2}{|}{} \\
\cline { 1 - 2 } Atributos biofísicos & 18.48 & \\
\cline { 1 - 2 } &
\end{tabular}

Los resultados de la Tabla 5 representan el comportamiento del indicador atributos estructurales, donde se observa que la sumatoria de las medias de los ítems es 7.00 , que se encuentra comprendida en la escala de (6-8), lo cual significa que los estudiantes expresan que los atributos estructurales de los espacios públicos de la Facultad de Arquitectura y Urbanismo, son adecuados, lo cual implica que el campus Capanique SL-02 de la Universidad Privada de Tacna, cumple con las condiciones fundamentales que exige la calidad en la formación de los estudiantes.

Tabla 5: Atributos estructurales

\begin{tabular}{|c|c|c|}
\hline Indicador & Media & Desv. típica \\
\hline Atributos estructurales & 3.50 & 0.39 \\
\hline Indicador & Sumatoria & \multicolumn{1}{|}{} \\
\cline { 1 - 2 } Atributos estructurales & 7.00 & \\
\cline { 1 - 2 }
\end{tabular}

Los resultados de la Tabla 6 representan el comportamiento del indicador atributos estéticos, donde se observa que la sumatoria de las medias de los ítems es 10.23 , que se encuentra comprendida en la escala de (7-12), lo cual significa que los estudiantes expresan que los atributos estéticos de los espacios públicos de la Facultad de Arquitectura y Urbanismo, son adecuados, lo cual implica que el campus Capanique SL-02 de la Universidad Privada de Tacna, cumple con las condiciones fundamentales que exige la calidad en la formación de los estudiantes.

Tabla 6: Atributos estéticos

\begin{tabular}{|c|c|c|}
\hline Indicador & Media & Desv. típica \\
\hline Atributos estéticos & 3.41 & 0.50 \\
\hline Indicador & Sumatoria & \multicolumn{1}{|}{} \\
\hline Atributos estéticos & 10.23 & \\
\cline { 1 - 2 }
\end{tabular}

Los resultados de la Tabla 7 representa el resumen del comportamiento de la variable espacios públicos, en los cuales se observa que la sumatoria de las tres dimensiones alcanza el valor de 44.42, ubicándose en el intervalo ( 43-63) lo cual significa que los estudiantes sostienen que los espacios públicos de la Facultad de Arquitectura y Urbanismo alcanzan el nivel de bueno, por lo tanto el Campus Capanique SL-02 de la Universidad Privada de Tacna, cumple con las condiciones fundamentales que exige la calidad en la formación de los estudiantes $(p<0.05)$.

Tabla 7: Resumen de espacios Públicos

\begin{tabular}{|r|c|c|}
\hline \multicolumn{1}{|c|}{ Dimensiones } & Media & Desv. Típica \\
\hline 1. Espacios públicos & 14.55 & 0.39 \\
\hline 2. Uso y apropiación de espacios & 11.39 & 0.50 \\
\hline 3. Valoración y calidad del paisaje & 18.48 & 0.35 \\
\hline TOTAL & 44.42 & 1.24 \\
\hline
\end{tabular}


Los resultados de la Tabla 8 representan el comportamiento del indicador utilización de espacio público en el ejercicio docente, donde se observa que la sumatoria de las medias de los ítems es 17.65, que se encuentra comprendida en la escala de (13-20), lo cual significa que los estudiantes expresan que en la Facultad de Arquitectura y Urbanismo se utiliza en forma adecuada los espacios públicos en la práctica docente, contribuyendo con la adecuada formación universitaria que se brinda en la Universidad Privada de Tacna.

Tabla 8: Utilización del espacio público en el ejercicio docente

\begin{tabular}{|c|c|c|}
\hline \multicolumn{1}{|c|}{ Indicador } & Media & Desv. típica \\
\hline $\begin{array}{c}\text { Espacio público en el } \\
\text { ejercicio docente }\end{array}$ & 4.41 & 0.43 \\
\hline \multicolumn{1}{|l|}{ Indicador } & Sumatoria & \multicolumn{1}{|}{} \\
\hline $\begin{array}{l}\text { Espacio público en el } \\
\text { ejercicio docente }\end{array}$ & 17.65 & \\
\cline { 1 - 2 }
\end{tabular}

Los resultados de la Tabla 9 representan el comportamiento del indicador formación humanística e integral, donde se observa que la sumatoria de las medias de los ítems es 8.05 , que se encuentra comprendida en la escala de (7-10), lo cual significa que los estudiantes expresan que en la Facultad de Arquitectura y Urbanismo se promueve y desarrolla el uso adecuado de los ambientes en las clases de paisajismo, demostrando de esta forma que la Universidad Privada de Tacna brinda un servicio educativo de calidad.

Tabla 9: Ambiente y clase de paisajismo

\begin{tabular}{|c|c|c|}
\hline Indicador & Media & Desv. típica \\
\hline Ambiente y clase de paisajismo & 4.02 & 0.50 \\
\hline Indicador & \multicolumn{2}{|c|}{ Sumatoria } \\
\hline Ambiente y clase de paisajismo & \multicolumn{2}{|c|}{8.05} \\
\hline
\end{tabular}

Los resultados de la Tabla 10 representan el comportamiento del indicador formación en base a competencias, donde se observa que la sumatoria de las medias de los ítems es 8.33 , que se encuentra comprendida en la escala de (7-10), lo cual significa que los estudiantes expresan que en la Facultad de Arquitectura y Urbanismo se promueve y desarrolla una adecuada formación en base a competencias, que contribuyen con la adecuada formación universitaria que se brinda en la Universidad Privada de Tacna.

Tabla 10: Formación en base a competencias

\begin{tabular}{|c|c|c|}
\hline Indicador & Media & Desv. típica \\
\hline Formación en base a competencias & 4.16 & 0.19 \\
\hline Indicador & \multicolumn{2}{|c|}{ Sumatoria } \\
\hline Formación en base a competencias & \multicolumn{2}{|c|}{8.33} \\
\hline
\end{tabular}

Los resultados de la Tabla 11 representan el comportamiento del indicador formación humanística e integral, donde se observa que la sumatoria de las medias de los ítems es 8.76, que se encuentra comprendida en la escala de (7-10), lo cual significa que los estudiantes expresan que en la Facultad de Arquitectura y Urbanismo se promueve y desarrolla una adecuada formación humanística e integral, que contribuyen con la adecuada formación universitaria que se brinda en la Universidad Privada de Tacna. 
Tabla 11: Formación humanística e integral

\begin{tabular}{|c|c|c|}
\hline Indicador & Media & Desv. típica \\
\hline Formación humanística e integral & 4.38 & 0.30 \\
\hline Indicador & \multicolumn{2}{|c|}{ Sumatoria } \\
\hline Formación humanística e integral & \multicolumn{2}{|c|}{8.76} \\
\hline
\end{tabular}

Los resultados de la Tabla 12 representan el comportamiento del indicador formación en innovación tecnológica, donde se observa que la sumatoria de las medias de los ítems es 9.03, que se encuentra comprendida en la escala de (7-10), lo cual significa que los estudiantes expresan que en la Facultad de Arquitectura y Urbanismo se promueve y desarrolla una adecuada formación en innovación tecnológica, que contribuyen con la adecuada formación universitaria que se brinda en la Universidad Privada de Tacna.

Tabla 12: Formación en innovación tecnológica

\begin{tabular}{|c|c|c|}
\hline Indicador & Media & Desv. típica \\
\hline Innovación tecnológica & 4.51 & 0.77 \\
\hline Indicador & \multicolumn{2}{|c|}{ Sumatoria } \\
\hline Innovación tecnológica & \multicolumn{2}{|c|}{9.03} \\
\hline
\end{tabular}

Los resultados de la Tabla 13 representan el comportamiento del indicador formación en responsabilidad social, donde se observa que la sumatoria de las medias de los ítems es 8.50 , que se encuentra comprendida en la escala de (7-10), lo cual significa que los estudiantes expresan que en la Facultad de Arquitectura y Urbanismo se promueve y desarrolla una adecuada formación en responsabilidad social, que contribuyen con la adecuada formación universitaria de la Universidad Privada de Tacna.

Tabla 13: Formación en responsabilidad social

\begin{tabular}{|c|c|c|}
\hline Indicador & Media & Desv. típica \\
\hline Responsabilidad social & 4.25 & 0.89 \\
\hline Indicador & \multicolumn{2}{|c|}{ Sumatoria } \\
\hline Responsabilidad social & \multicolumn{2}{|c|}{8.50} \\
\hline
\end{tabular}

Los resultados de la Tabla 14 representan el comportamiento del indicador formación en desarrollo sostenible, donde se observa que la sumatoria de las medias de los ítems es 9.10, que se encuentra comprendida en la escala de (7-10), lo cual significa que los estudiantes expresan que en la Facultad de Arquitectura y Urbanismo se promueve y desarrolla una adecuada formación con base en el enfoque de desarrollo sostenible que contribuyen con la adecuada formación universitaria de la Universidad Privada de Tacna.

Tabla 14: Formación para el Desarrollo Sostenible

\begin{tabular}{|c|c|c|}
\hline Indicador & Media & Desv. típica \\
\hline Desarrollo sostenible & 4.55 & 0.52 \\
\hline Indicador & \multicolumn{2}{|c|}{ Sumatoria } \\
\hline Desarrollo sostenible & \multicolumn{2}{|c|}{9.10} \\
\hline
\end{tabular}

Los resultados de la Tabla 15 representan el comportamiento del indicador formación para el mundo global, donde se observa que la sumatoria de las medias de los ítems es 9.45, que se encuentra 
comprendida en la escala de (7-10), lo cual significa que los estudiantes expresan que en la Facultad de Arquitectura y Urbanismo se desarrolla una formación para el mundo que contribuyen con la adecuada formación universitaria de la Universidad Privada de Tacna.

Tabla 15: Formación para el mundo globalizado

\begin{tabular}{|c|c|c|}
\hline Indicador & Media & Desv. típica \\
\hline $\begin{array}{c}\text { Formación para el mundo } \\
\text { global }\end{array}$ & 4.72 & 0.61 \\
\hline $\begin{array}{c}\text { Indicador } \\
\begin{array}{c}\text { Formación para el mundo } \\
\text { global }\end{array}\end{array}$ & \multicolumn{2}{|c|}{ Sumatoria } \\
\hline
\end{tabular}

Los resultados de la Tabla 16 representan el comportamiento del indicador áreas verdes, donde se observa que la sumatoria de las medias de los ítems es 20.8, que se encuentra comprendida en la escala de (16-25), lo cual significa que los estudiantes expresan que en la Facultad de Arquitectura y Urbanismo se dispone de áreas verdes adecuadas que contribuyen con la adecuada formación universitaria de la Universidad Privada de Tacna.

Tabla 16: Importancia de las áreas verdes

\begin{tabular}{|c|c|c|}
\hline Indicador & Media & Desv. típica \\
\hline Áreas verdes & 4.16 & 1.38 \\
\hline Indicador & \multicolumn{2}{|c|}{ Sumatoria } \\
\hline Áreas verdes & \multicolumn{2}{|c|}{20.8} \\
\hline
\end{tabular}

Los resultados de la Tabla 17 representa el resumen del comportamiento de la variable formación universitaria, a través de los resultados del análisis de cada dimensión, en ese sentido se observa que la sumatoria de las cuatro dimensiones alcanza el valor de 64.03, ubicándose en el intervalo ( $52-80$ ) lo cual significa que los estudiantes expresan que en la facultad de Arquitectura y Urbanismo se brinda una buena formación universitaria, demostrando de esta forma que en la Universidad Privada de Tacna la calidad en formación profesional es adecuada.

Tabla 17: Resumen formación universitaria

\begin{tabular}{|l|c|c|}
\hline Dimensiones & Media & Desv. Típica \\
\hline Aprendizaje vivencial & 17.65 & 0.43 \\
\hline Conciencia y formación ambiental & 8.05 & 0.50 \\
\hline Calidad universitaria & 17.53 & 0.89 \\
\hline Valoración y Calidad del paisaje & 20.80 & 1.38 \\
\hline Total & 64.03 & 3.20 \\
\hline
\end{tabular}

Con un nivel de confianza del 95\%, se determina que existe evidencia de la relación significativa entre la organización espacial y la formación universitaria de los estudiantes de la Facultad de Arquitectura y Urbanismo de la Universidad Privada de Tacna ( $p$ : 0.00). Existe relación directa entre el uso y apropiación del espacio, valoración y calidad del paisaje y la formación universitaria de los estudiantes de la Facultad de Arquitectura y Urbanismo de la Universidad ( $r: 0.746$ ) 


\section{DISCUSIÓN}

Según Borja (2003) que sostiene acerca de los espacios públicos dentro de la ciudad, guardan correspondencia entre Universidad y Ciudad, de esta forma. Si los espacios públicos son vitales para el desarrollo de la ciudad y del mejoramiento de la calidad de vida del ciudadano, en la misma medida también los espacios públicos en una Universidad también son vitales para la calidad de la formación de los estudiantes. El impacto de los espacios públicos es muy importante en las políticas de gestión de las universidades, considerando que tiene influencia en la calidad de formación universitaria de los estudiantes. De la buena gestión de los espacios públicos en las Facultades de las universidades, dependerá la calidad de la formación de profesionales íntegros de alto nivel, que reciben una formación integral más allá del mero conocimiento académico.

La investigación permite confirmar que los espacios públicos tienen influencia en la formación universitaria de los estudiantes de la Facultad de Arquitectura. En ese sentido la teoría de Borja acredita la importancia que tiene la adecuada implementación y desarrollo de los espacios públicos de los Campus universitarios en la formación integral de los estudiantes.

Los espacios públicos del Campus LS-02 de la Facultad de Arquitectura y Urbanismo tiene influencia significativa en la formación universitaria de los estudiantes del primer al décimo ciclo, en la Universidad Privada de Tacna. A nivel de Facultad el estudio, ha permitido establecer que existe un nivel adecuado de implementación y desarrollo de los espacios públicos en el Campus SL-02, así como en la misma medida también existe una adecuada calidad universitaria en la Facultad de Arquitectura y urbanismo, siendo en suma buena la formación universitaria impartida en la Facultad. La óptima formación universitaria depende en gran medida de las políticas de atención y desarrollo de los espacios públicos de cada una de las Facultades que forman profesionales en la Universidad Privada de Tacna, considerando que son componentes básicos del modelo de calidad el diseño e implementación y uso de los Campus como espacios públicos que aseguran la calidad en la formación de profesionales en las Facultades de Arquitectura y Urbanismo. Es importante recalcar, que el resultado obtenido del análisis, nos sitúa en un estándar medio, en comparación a las grandes universidades de la capital, convirtiéndose así esta investigación en un aporte para incentivar a los directivos correspondientes, en potenciar los espacios públicos actuales. El uso y apropiación de los espacios públicos es uno de los aspectos que forma parte de la acreditación de las facultades, por lo que es responsabilidad de directivos, docentes y estudiantes brindar la importancia debida para lograr la atención y priorización de la Asamblea Universitaria.

\section{REFERENCIAS BIBLIOGRÁFICAS}

Borja, J. (2000). El espacio público ciudad y ciudadanía.

Bur, J. (2011). Aprendizaje vivencial en el aula universitaria.

Burneo, L (2011). Construcción de la ciudadanía mediante el uso cotidiano del espacio público.

Castro, M. y Llanes, J. (2012). Habilidades para la vida en estudiantes universitarios.

Campos, P. (2006). Memoria y Proyección del Espacio Urbano Universitario en España.

Consejo de Europa (2006). Convenio Europeo del Paisaje. Florencia: Consejo de Europa.

Dirección General de Territorio y Paisaje (2012). Plan de Acción territorial de la Huerta en Valencia.

España

El observatorio del paisaje (2006). Objetivos de calidad paisajista de Cataluña. España

Figueroa, C (2014). Las áreas verdes en relación con la enseñanza - aprendizaje de las Ciencias Naturales y educación ambiental.

Gehl, J (2004). La Humanización del Espacio Urbano - La vida social entre los Edificios.

Giddens, A (1995). La constitución de la sociedad. Bases para la teoría de la estructuración. 
Gomera, A (2008). La Conciencia ambiental como herramienta para la educación ambiental: Conclusiones y reflexiones de un estudio en el ámbito universitario

Gonzales, N. (2013). La Trilogía de la Arquitectura. Infraestructura. Calidad. Educación. La importancia de la Infraestructura como factor Determinante en el Aprendizaje del Estudiante de Arquitectura.

Gonzales, P (2016). Uso y apropiación del espacio público para las actividades sociales en el sector cc, sub sector 10 y 11 de Huancayo.

Gussinyer, G (2012). Notas para el concepto de espacio en la arquitectura precolombina de Mesoamérica. Universidad de Barcelona.

Herrán, A. de la y Álvarez, N. (2010). Para qué enseñar: Significado y sentido de la formación universitaria.

Jover, G., y Gozalvez, V. (2012) La universidad como espacio público: un análisis a partir de dos debates en torno al pragmatismo. Bordon 64(03), 39-52.

Marín, L (2016). Usos del espacio público y su influencia en la dinámica social del sector La Esperanza del distrito de Chilca.

Onu, Habitat. Guía de diseño del espacio público seguro, incluyente y sustentable - Programa de las Naciones Unidas para los asentamientos humanos, Secretaria de Desarrollo Social. México. SEDESOL

Palermo, U. (2009). Cuaderno 30/Cuaderno del Centro de Estudios en Diseño y Comunicación.

Palomino, D (2010). Regeneración del Parque la Colonia. Ley 57 en Hermosillo, Sonora

Paramo, Burbano, P, A (2014). Los usos y la apropiación del espacio urbano para el fortalecimiento de la democracia.

Ramos de Robles, L. (2016) El valor Educativo de las áreas verdes: Educarnos. Recuperado de https://revistaeducarnos.com

Salcedo, JC. (2001). La Pérdida del Espacio Universitario en el Centro Histórico de Cáceres.

Sedesol, (2016). Documento diagnóstico de rescate de espacios públicos.

UPT. (2012). Guía Metodológica para el procedimiento del diseño curricular

Servicio de Evaluación Ambiental (2013). Valor paisajista en el SEIA - Gobierno de Chile.

Verdegen. (2017). Generación Verde. CDMX. Recuperado de https://generacionverde.com 